\title{
Excitation of VLF and ELF Radio Waves by a Horizontal Magnetic Dipole ${ }^{1,2}$
}

\author{
Janis Galejs
}

\author{
(August 29, 1960; revised November 10, 1960)
}

The VLF and ELF modes excited by a horizontal magnetic dipole (vertical loop) in the spherical shell between a finitely conducting earth and an isotropic sharply bounded ionosphere are shown to have a nearly transverse magnetic character. The modes are similar to those of a vertical electric dipole. With the exception of the zero order mode, the propagating modes excited by the magnetic dipole are of slightly higher amplitudes, provided that the far fields of the horizontal magnetic and vertical electric dipoles are equal over flat earth in the absence of ionosphere.

The transient fields generated by a current step in the magnetic dipole are in the first approximation similar to the fields generated by a current impulse in a vertical electric dipole. Response of the zero order mode of the magnetic dipole has been calculated.

\section{Introduction}

The mode theory of VLF transmissions has been developed by Watson [1919], Budden [1953], Schumann [1954], and Wait [1957, 1960a, 1960b] for excitation by vertical and horizontal electric dipoles and also by vertical magnetic dipoles (horizontal loops). Additional references on this subject have been listed by Wait [1960a, 1960b].

The reciprocity theorem has been found to be useful for relating the fields of a horizontal electric dipole to fields of vertical electric and magnetic dipoles [Wait, 1960a]. The reciprocity relations may be also applied to relate the fields due to horizontal magnetic dipole excitation to known fields due to vertical electric and magnetic dipoles.

\section{Reciprocity Relations}

The reciprocity theorem states that the voltage $V_{2}$ induced in antenna 2 by current $I_{1}$ of antenna 1 is the same as the voltage $V_{1}$ induced in antenna 1 by an identical current $I_{2}$ flowing in antenna 2 .

A vertical electric $(V E)$ dipole of length $d s$ at $z_{0}^{\prime}=z_{t}$ and a horizontal magnetic $(H M)$ dipole parallel to the $x$-axis (or a vertical loop of area $d a$ in the $y, z$ plane) at $z_{0}=z_{r}$ are shown in figure 1 , where subscripts $r$ and $t$ refer to receiver and transmitter coordinates respectively. The only nonzero $H$ component generated by the $V E$ dipole is $H_{\phi^{\prime}}$. The magnitude of the voltage induced in the $H M$ dipole $V^{h m}$ is maximum if $H_{\phi^{\prime}}$ is parallel to the $x$-axis,

1 Contribution from Applied Research Laboratory of Sylvania Electronic Systems, Waltham, Mass.

2 The research reported in this paper was supported by a contract of the Office of Naval Research. which occurs for $\phi=\frac{\pi}{2}$ and $\frac{3 \pi}{2}$. For $e^{i \omega t}$ variation of the fields.

$$
V^{h m}=-i \omega \mu_{0} H_{\phi^{\prime}}^{v e}\left(z_{0}^{\prime}=z_{t}, z_{0}=z_{n}\right) \sin \phi d a,
$$

where the superscripts $h m$ and ve refer to horizontal magnetic and vertical electric dipoles respectively. With the same current applied to the $H M$ dipole only the vertical electric field $E_{z}$ will contribute to $V^{v e}$ and

$$
V^{v e}=E_{z}^{h m}\left(z_{0}=z_{t}, z_{0}^{\prime}=z_{r}\right) d s .
$$

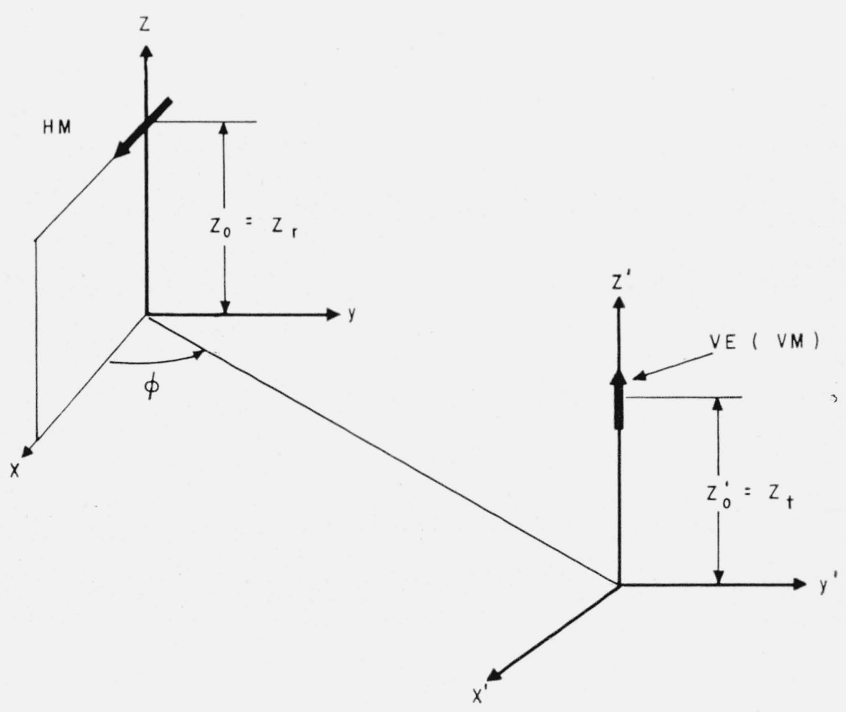

Figure-1. Coordinate systems for defining reciprocity relations. 
Equating (1) and (2) results in

$$
\begin{aligned}
E_{z}^{h m}\left(z_{0}=z_{t}, z_{0}^{\prime}\right. & \left.=z_{r}\right) \\
& =-i \omega \mu_{0} \sin \phi H_{\phi^{\prime}}^{v e}\left(z_{0}^{\prime}=z_{t}, z_{0}=z_{r}\right) d a / d s .
\end{aligned}
$$

A vertical magnetic $(V M)$ dipole (or a horizontal loop of area $d a^{v m}$ parallel to the $x^{\prime}, y^{\prime}$ plane) at $z_{0}^{\prime}=z_{t}$ and a horizontal magnetic $(H M)$ dipole parallel to the $x$-axis (or a vertical loop of area $d a^{h m}$ in the $y, z$ plane) at $z_{0}=z_{r}$ may be considered in the geometry shown in figure 1 . The only nonzero $H$ component generated by the $V M$ dipole is $H_{\rho^{\prime}}$. The magnitude of the voltage induced in the $H M$ dipole $V^{h m}$ is maximum, if $H_{\rho^{\prime}}$ is parallel to the $x$-axis, which occurs for $\phi=0$ or $\pi$. Hence

$$
V^{h m}=i \omega \mu_{i} H_{\rho^{\prime}}^{v m}\left(z_{0}^{\prime}=z_{t}, z_{0}=z_{r}\right) \cos \phi d a^{h m}
$$

where the superscript $\mathrm{vm}$ refers to the vertical magnetic dipole. With the same current applied to the $H M$ dipole only the vertical magnetic field $H_{z}$ will contribute to $\dot{V}^{v m}$ and

$$
V^{v m}=-i \omega \mu_{0} H_{z}^{h m}\left(z_{0}=z_{t}, z_{0}^{\prime}=z_{r}\right) d a^{v m} .
$$

Equating (4) and (5) gives for $d a^{v m}=d a^{h m}$

$$
H_{z}^{h m}\left(z_{0}=z_{t}, z_{0}^{\prime}=z_{r}\right)=-\cos \phi H_{\rho^{\prime}}^{v m}\left(z_{0}^{\prime}=z_{t}, z_{0}=z_{r}\right) .
$$

For spherical coordinates the $z$ component is replaced by $r$ component and the $\rho$ component by the $\theta$ component of the fields.

The reciprocity relations apply to dipoles in the presence of any linear media. The fields of dipoles above plane earth derived by solving the wave equation should also satisfy (3) and (6). Thus (27) and (116) of Norton [1937], which have been derived for unit dipoles, check the validity of (3). Some algebra is involved in verifying (6) from (35.1) of Sommerfeld [1949] and from (2) to (6), (17) to (19), and (30) of Norton [1937].

\section{Fields of the Horizontal Magnetic Dipole}

The dipole fields will be examined with the aid of reciprocity relations in an idealized spherical shell that is bounded by a homogeneous earth of radius $a$, conductivity $\sigma_{g}$ and dielectric constant $\epsilon_{g}$ and by a homogeneous ionosphere of radius $(a+h)$, conductivity $\sigma_{i}$ and dielectric constant $\epsilon_{i}$. 'I he above model neglects the effects of the earth's magnetic field.

The vertical electric field $E_{r}^{h m}$ is computed by (3) applying (5.1), (6.16) of Wait [1960a] and using the relation

$\frac{d}{d \theta} P_{\nu}(-\cos \theta)$

$$
=\nu\left[\cos \theta P_{\nu}(-\cos \theta)+P_{\nu-1}(-\cos \theta)\right] / \sin \theta .
$$

This results in

$$
\begin{array}{r}
E_{r}^{h m}=\frac{I(d a) i \omega \mu_{0} \sin \phi}{2 h r_{r}} \sum_{n=0}^{\infty} f_{n}\left(z_{t}\right) f_{n}\left(z_{r}\right) \cdot \frac{\delta_{n}}{\sin \nu \pi} \frac{\nu}{\sin \theta} \\
{\left[\cos \theta P_{\nu}(-\cos \theta)+P_{\nu-1}(-\cos \theta)\right] .}
\end{array}
$$

The vertical magnetic field $H_{r}^{h m}$ is computed by (6) applying (9.1), (9.2) of Wait [1960a] and using the relation (7) as

$$
\begin{gathered}
H_{r}^{h m}=-\frac{I(d a) \omega \mu_{0} \cos \phi}{2 k h r_{t} r_{r} \eta} \sum_{m=1}^{\infty} \frac{\delta_{m} f_{m}\left(z_{r}\right) \mu}{\sin \mu \pi \sin \theta} \\
\quad\left[f_{m}\left(z_{t}\right)+r_{t} \frac{d}{d z_{t}} f_{m}\left(z_{t}\right)\right] \\
\quad\left[\cos \theta P_{\mu}(-\cos \theta)+P_{\mu-1}(-\cos \theta)\right] .
\end{gathered}
$$

Using the second order representation of the radial functions, applying (6.9), (6.17), (9.3), and (9.4) of Wait [1960a] yields

$$
\begin{gathered}
2 f_{q}(z)=e^{i k C_{q} z}\left[R_{g}^{q}\left(C_{q}\right)\right]^{-1 / 2}+e^{-i k C} q^{z}\left[R_{g}^{q}\left(C_{q}\right)\right]^{1 / 2} \\
\delta_{q}=\left(1 \pm \frac{\sin 2 k h C_{q}}{2 k h C_{q}}\right)^{-1}
\end{gathered}
$$

where $q$ is an integer equal to either $n$ or $m$ and where

$$
\begin{gathered}
R_{p}^{n}\left(C_{n}\right)=\frac{n_{p} C_{n}-C_{p}}{n_{p} C_{n}+C_{p}} \\
R_{p}^{m}\left(C_{m}\right)=\frac{C_{m}-n_{p} C_{p}}{C_{m}+n_{p} C_{p}} \\
C_{q}=\sqrt{1-S_{q}^{2}}, \\
C_{p}=\sqrt{1-\left(S_{q} / n_{p}\right)^{2}}, \\
S_{q}=\frac{\rho-0.5}{k a} .
\end{gathered}
$$

The plus sign of (11) should be used with $q=n$, while the minus sign is appropriate for $q=m$. In (16) $\rho$ is a complex number, which is equal to $\nu$ of (8) or equal to $\mu$ of $(9), k=\omega / c, c=$ velocity of light. The refractive index $n$ of the boundary medium characterized by subscript $p$ is

$$
n_{p}^{2}=\left(\sigma_{p}+i \omega \epsilon_{p}\right) / \epsilon_{0}
$$

where $\sigma_{p}$ and $\epsilon_{p}$ are the conductivity and permittivity of the boundary medium and where $\epsilon_{0}$ is the permittivity of free space $\left(\epsilon_{0}=(36 \pi)^{-1} 10^{-9}\right.$ farad/ meter). The boundary medium can be either ground $(p=g)$ or ionosphere $(p=i)$. The refractive index of ground is

$$
n_{g}^{2} \approx \sigma_{g} /\left(i \omega \epsilon_{0}\right)
$$


where $\sigma_{g}$ is the ground conductivity. The refractive index of the ionosphere is

$$
n_{i}^{2}=1+\sigma_{i} /\left(i \omega \epsilon_{0}\right) .
$$

The ionospheric conductivity $\sigma_{i}$ is defined by

$$
\sigma_{i}=\frac{\epsilon_{0} \omega_{p}^{2}}{\underline{\nu}}
$$

where $\underline{\nu}$ is the electron collision frequency in the ionosphere and $\omega_{p}$ is the plasma frequency $\left(\omega_{p}^{2}=3180\right.$ $N, N=$ number of electrons per meter $\left.{ }^{3}\right) . \quad{ }^{2} C_{n}$ and $C_{m}$ are roots of the modal equations

$$
R_{i}^{q}\left(C_{q}\right) R_{g}^{q}\left(C_{q}\right)=\exp \left(+2 i k h C_{q}\right) .
$$

$S_{q}$ that is related to $C_{q}$ by (14) has a magnitude of approximately unity and a small imaginary part for propagating modes of low attenuation. With $S_{n}$ and $S_{m}$ determined from (14), $\nu$ of (8) and $\mu$ of (9) follow from (16). This completes the formal specification of the fields $E_{r}^{h m}$ and $H_{r}^{h m}$.

The expressions for $E_{r}^{h m}$ and $H_{r}^{h m}$ may be simplified by introducing further approximations. The consideration will be restricted to cases where

$$
\begin{gathered}
r_{r} \approx r_{t} \approx a, \\
k C_{q} z_{r} \ll 1, \\
k C_{q} z_{t} \ll 1, \\
n_{g} \gg 1 .
\end{gathered}
$$

This gives

$$
\begin{gathered}
R_{g}^{n}\left(C_{n}\right) \approx 1-\frac{2}{n_{g} C_{n}} \approx 1, \\
R_{g}^{m}\left(C_{m}\right) \approx-1+\frac{2 C_{m}}{n_{g} C_{g}} \approx-1, \\
f_{n}(z) \approx 1+\frac{1}{8 n_{g}^{2} C_{n}^{2}} \approx 1, \\
f_{m}(z) \approx C_{m} /\left(i n_{g} C_{g}\right) \approx C_{m} /\left(i n_{g}\right), \\
d f_{m}(z) / d z=i k n_{g} C_{g} f_{m}(z) \approx i k n_{g} f_{m}(z) .
\end{gathered}
$$

Approximating the Legendre functions by [Watson, 1919 ; Bremmer, 1949]

$P_{\rho}(-\cos \theta) \approx(2 \pi \rho \sin \theta)^{-0.5} \exp \left[i\left(\rho+\frac{1}{2}\right)(\pi-\theta)-i \pi / 4\right]$

results in

$$
\begin{array}{r}
E_{r}^{h m}=-\sin \phi E_{0}^{h m} \sqrt{\frac{d / a}{\sin (d / a)}} \frac{\sqrt{d / \lambda}}{h / \lambda} e^{i \frac{2 \pi d}{\lambda}-i \frac{\pi}{4}} \\
\cdot \sum_{n=0}^{\infty} \delta_{n} S_{n}^{0.5} e^{-i \frac{2 \pi d}{\lambda} S_{n}},
\end{array}
$$

$$
\begin{aligned}
& H_{r}^{h m}=-\cos \phi \frac{E_{0}^{h m}}{\eta n_{g}} \sqrt{\frac{d / a}{\sin (d / a)}} \frac{\sqrt{d / \lambda}}{h / \lambda} e^{i \frac{2 \pi d}{\lambda}-i \frac{\pi}{4}} \\
& \cdot \sum_{m=1}^{\infty} \delta_{m}\left(1-S_{m}^{2}\right) S_{m}^{0.5} e^{-i \frac{2 \pi d}{\lambda} S_{m}},
\end{aligned}
$$

where the distance along the curved earth is

and where

$$
d=a \theta,
$$

$$
E_{0}^{h m}=\frac{2 \pi I(d a) \eta}{\lambda^{2} d} e^{-i \frac{2 \pi d}{\lambda}}
$$

is the vertical electric field of the source at a distance $d$ in the direction of maximum intensity on a perrectly conducting plane earth.

\section{Comparison of Harmonically Excited Dipole Fields}

The fields of the horizontal magnetic dipole $E_{r}^{h m}$ and $H_{T}^{h m}$ will be compared first with the corresponding field components of the horizontal electric dipole. It follows from (9.37) and (9.42) of Wait [1960a] that for the $n^{\text {th }}$ and the $m^{\text {th }}$ mode

$$
\begin{aligned}
& \left.\frac{E_{r}^{h m}}{E_{0}^{h m}}\right|_{n}=-\left.n_{g} \tan \phi \frac{E_{r}^{h e}}{E_{0}^{v e}}\right|_{n} \\
& \left.\frac{H_{r}^{h m}}{E_{0}^{h m}}\right|_{m}=\left.n_{g} \cot \phi \frac{H_{r}^{h e}}{E_{0}^{v e}}\right|_{m}
\end{aligned}
$$

where the superscripts he and ve designate field components of horizontal electric and of vertical electric dipoles respectively. $\quad E_{0}^{h m}$ is defined in (35), $E_{0}^{v e}$ is the corresponding expression of the vertical electric dipole defined by (6.25) or (9.38) of Wait [1960a]. The approximation $C_{g} \approx 1$ was used in the expressions for $E_{r}^{\text {he }}$ and $H_{r}^{\text {he }}$ in order to make them consistent with the derivation leading to (32) and (33). $\quad C_{g} \approx 1$ constitutes a better approximation than $f_{n}(z) \approx 1$ and the assumption $f_{n}(z) \approx 1$ [Wait, 1960a] should be followed by $C_{g} \approx 1$.

Comparison of (32) and (33) shows that $\eta H_{r}^{h m}$ is several orders of magnitude smaller than $E_{r}^{h m}$ because

and because

$$
n_{g}^{-1} \ll 1
$$

$$
1-S_{m}^{2} \ll 1
$$

for propagating modes of low attenuation. The $H_{r}^{h m}$ fields of a given mode are proportional to $n_{g}^{-1} \sim \sqrt{\omega / \sigma_{g}}$. The fields are decreased with decreasing frequency $\omega$ or increasing ground conductivity $\sigma_{g}$. Similar comments apply to the transverse electric (TE) field components that may be derived from $H_{r}$. The $T E$ fields will not be considered in more detail.

The component $E_{r}^{h m}$ that is associated with transverse magnetic $(T M)$ fields may be compared with the corresponding component $\dot{E}_{r}^{v e}$ of a vertical elec- 
tric dipole using an expression of the $E_{r}^{v e}$ fields derived under similar assumptions [Wait, 1957]. Thus

$\left.E_{r}^{h m}\right|_{n}=-\left.\frac{\sin \phi E_{0}^{h m}}{S_{n} E_{0}^{v e}} E_{r}^{v e}\right|_{n}=\left.\frac{i k I^{h m} d a}{S_{n} I^{v e} d s} \sin \phi E_{r}^{v e}\right|_{n}$.

Provided that the fields of the two antennas are the same in the given direction over a perfectly conducting plane earth $\left(\sin \phi E_{0}^{h m}=E_{0}^{v e}\right)$, (40) may be simplified to

$$
\left.\frac{E_{r}^{v e}}{E_{r}^{h m}}\right|_{n}=-S_{n}
$$

Substituting the reciprocity relation (3) in (40) follows that $\left(E_{\tau}^{v e}\right) /\left(\eta H_{\phi}^{v e}\right)$ also satisfy (41). The latter ratio, obtained from (13), (15), and (16) of Wait [1960b], when used in conjunction with (3) provides an alternate way of obtaining (40) and subsequently (41).

A more exact treatment of the horizontal magnetic dipole fields cannot be expected to lessen the importance of $T M$ relative to $T E$ field components. A better approximation to the $T M$ fields of the horizontal magnetic dipole may be worked out from the exact ratio $E_{r}^{v e} / E_{r}^{h m}$ derived in the appendix. As long as the source and observation point are at equal radii $r$ the ratio involves only the azimuthal $\theta$ functions explicitly. However, the accuracy of the roots $S_{n}$ and of $E_{r}^{v e}$ depends on the approximations to the azimuthal and radial functions.

Numerical values of $S_{n}$ are available from mode calculations of the vertical electric dipole [Howe and Wait, 1957]. For $\sigma_{g}=\infty$ the values are as follows:

\begin{tabular}{|c|c|c|c|}
\hline $\begin{array}{c}h / \lambda \\
\pi \nu c /\left(\omega_{p}^{2} h\right)\end{array}$ & $\begin{array}{l}3.5 \\
0.1\end{array}$ & $\begin{array}{r}3.5 \\
0.01\end{array}$ & $\begin{array}{c}7 \\
0.1\end{array}$ \\
\hline $\begin{array}{l}1 \\
2 \\
2 \\
3 \\
4\end{array}$ & $\begin{array}{l}0.9975-i 3 \times 10^{-4} \\
.98-i 3 \times 10^{-3} \\
.93-i 0.01 \\
.87-i 0.02\end{array}$ & $\begin{array}{l}0.997-i 10^{-3} \\
.97-i 8 \times 10^{-3} \\
.91-i 9 \times 10^{-3} \\
.85-i 0.01\end{array}$ & $\begin{array}{l}0.995-i 6 \times 10^{-5} \\
.992-i 4 \times 10^{-4} \\
.98-i 10^{-\mathbf{3}} \\
.985-i 2 \times 10^{-8}\end{array}$ \\
\hline
\end{tabular}

The $n=0$ mode is severely attenuated for frequencies above a few kilocycles [Wait, 1957]. In the lower frequency range the first order perturbation solution of the modal equation may be used [Schumann, 1954], which gives

$C_{n}=\frac{\omega_{n}}{\omega}+\frac{i c}{h \omega C_{n}}\left[\frac{1}{n_{g}} \sqrt{1-\frac{1-C_{n}^{2}}{n_{g}^{2}}}+\frac{1}{n_{i}} \sqrt{1-\frac{1-C_{n}^{2}}{n_{i}^{2}}}\right]$.

The cutoff frequency of the $n$th mode is

$$
\omega_{n}=\frac{n \pi c}{h} .
$$

The perturbation method applies for $n \neq 0$ if $C_{n} \approx \omega_{n} / \omega$. Substituting this for $C_{n}^{-1}$ in the right-hand side of
(42) and expanding the square root expressions

$$
C_{n} \approx \frac{\frac{\omega}{\omega}+\frac{i_{n} c}{h \omega_{n}}\left[\left(\frac{1}{n_{g}}+\frac{1}{n_{i}}\right)-\frac{1}{2}\left(\frac{1}{n_{g}^{3}}+\frac{1}{n_{i}^{3}}\right)\right]}{1-\frac{i c}{2 h \omega}\left(\frac{1}{n_{g}^{3}}+\frac{1}{n_{i}^{3}}\right)}
$$

The latter expression may be also obtained by solving (42) as a quadratic and by subsequently ignoring the second order perturbation. Applying (14), simplifies for $n=0$ and $\left|n_{g}\right|,\left|n_{i}\right| \gg 1$ to

$$
S_{0}=\sqrt{1+\frac{c}{h i \omega}\left[\frac{1}{n_{g}}+\frac{1}{n_{i}}\right]}
$$

For $n_{g} \gg n_{i}$ and $\sigma_{i}=1.2 \times 10^{-6}\left(\omega_{p}^{2} / \nu=1.35 \times 10^{5}\right) \quad S_{0}$ has the following values

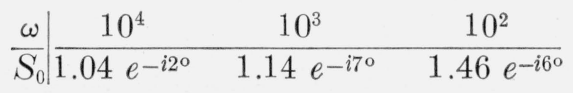

The above tabulations show that $\left|S_{n}\right|$ or the ratio $\left|E_{r}^{v e}\right| E_{r}^{h m} \mid$ are decreased with increasing mode index $n$ and, with the exception of $S_{0}$, also with decreasing frequency. The phase angle between the propagating modes of the two dipoles is negligible. The largest difference between the fields of the two dipoles may be expected for the lowest propagating frequencies or near the mode cutoff of $n \neq 0$ modes. The values of $S_{n}$ near the mode cutoff may be determined from an approximate solution of the modal equation. The first order perturbation solution is applicable only to the lower order modes. A different approximate solution is obtained by observing that $S_{n}$ is small near the cutoff $\left(S_{n}=1 /(2 \mathrm{ka})\right.$ at the mode cutoff for perfectly conducting ground and ionosphere according to Schumann [1954]). Hence one may look for solutions of the modal equation of the form

$$
C_{n}=\sqrt{1-S_{n}^{2}}=1-\Delta_{n}
$$

where $\left|\Delta_{n}\right| \ll 1$. Substituting (46) in (12), letting $p=i$ and defining

$$
L=\frac{\epsilon_{0} \omega}{\sigma_{i}}=\frac{\omega \nu}{\omega_{p}^{2}}
$$

results after neglecting terms with $\Delta_{n}^{2}$ and higher powers of $\Delta_{n}$ in

$$
R_{i}^{n}=i(2 L-i-2 \sqrt{L} \sqrt{L-i})\left(1-\frac{2 \Delta_{n} \sqrt{L}}{\sqrt{L-i}}\right)=L_{1}+\Delta_{n} L_{2} .
$$

Considering $n_{g}^{2} \gg 1$, substituting (46) in (12) and letting $p=g$ gives

$$
R_{g}^{n} \approx 1-2 n_{g}^{-1} e^{i \pi / 4}\left(1+\Delta_{n}\right)=G_{1}+\Delta_{n} G_{2} .
$$


The solution of the modal equation (21) becomes

$$
\Delta_{n}=\frac{2 i(k h-\pi n)-\ln L_{1} G_{1}}{2 i k h+\frac{L_{2}}{L_{1}}+\frac{G_{2}}{G_{1}}} .
$$

It is simplified at the cutoff of the respective mode by applying (43) to

$$
\Delta_{n}=-\frac{\ln L_{1} G_{1}}{2 i n \pi+\frac{L_{2}}{L_{1}}+\frac{G_{2}}{G_{1}}} .
$$

'The calculated curves of $\left|S_{n}\right|$ and of its argument are depicted in figures 2 and 3 for infinite ground conductivity. $\left|S_{n}\right|$ is smallest for small values of $I=\pi c \underline{\nu} /\left(h \omega_{p}^{2}\right)$ (or for large ionospheric conductivities) and for the higher modes (higher cutoff frequencies $\left.\omega_{n}=n \pi c / h\right)$. The argument of $S_{n}$ exhibits a relatively small variation with $n$. The increase of $\left|S_{n}\right|$ and of $\arg S_{n}$ with increasing $I$ signifies the increase of mode attenuation with decreasing ionospheric conductivity. The increase of $\left|S_{n}\right|$ from the $\sigma_{g}=\infty$ to the $\sigma_{g}=0.07 / h_{k m}$ curve $\left(\sigma_{g}=10^{-3} \mathrm{mho} / \mathrm{m}\right.$ for $h=70$ $\mathrm{km}$ ) is less than 20 percent for the $n=1$ mode in figure 4. This relative increase is even smaller for the higher $n$ values.

The amplitude ratio between electric and magnetic dipole fields in (41) will be larger for propagating modes than near the mode cutoff. The cutoff ratio is larger than 0.25 for the $n=1$ mode and larger than

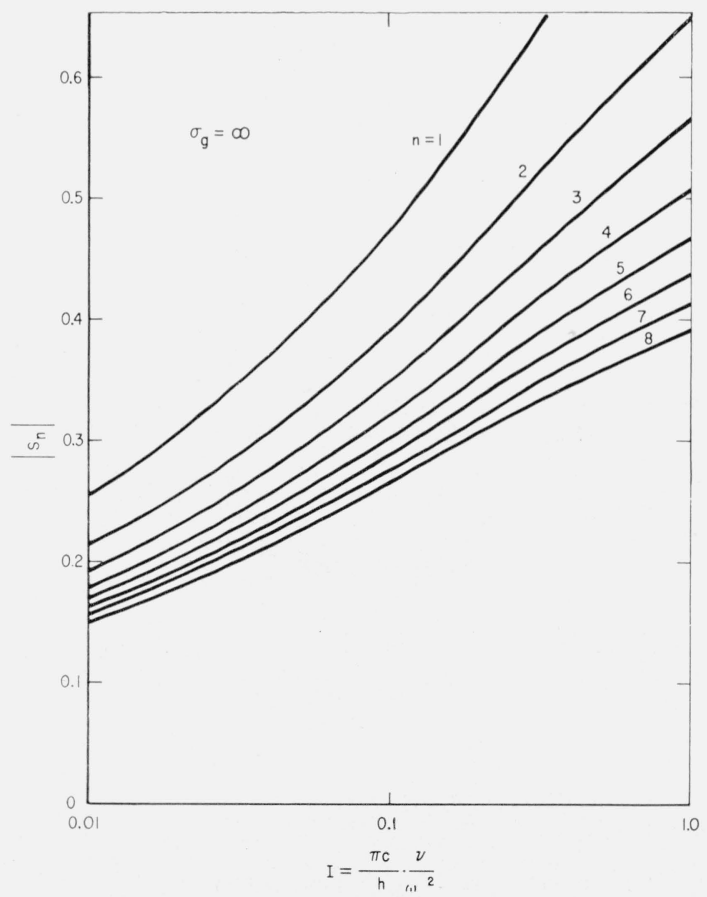

Figure 2. Magnitude of the constant $\mathrm{S}_{\mathrm{n}}$ near mode cutoff for perfectly conducting ground.
0.15 for the first 8 modes with $I>0.01$ (or for $\sigma_{i} \geq 1.2 \times 10^{-5}$ with $h=70 \mathrm{~km}$ ). It may be noted that this comparison is made for dipoles that exhibit equal far fields above a perfectly conducting plane ground in the absence of ionosphere. The knowledge of the above amplitude ratios would enter in a comparison of antenna radiation efficiencies. However, no such calculations have been attempted in this paper.

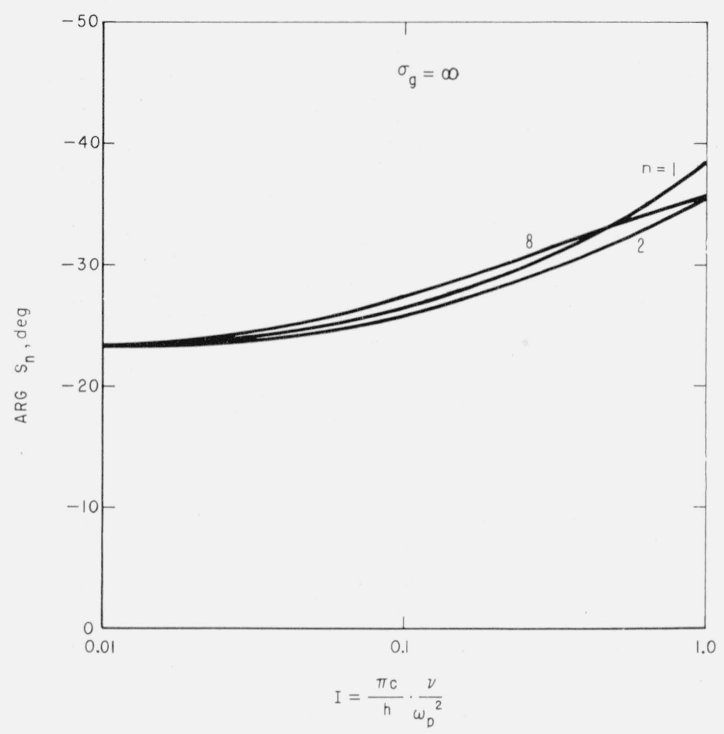

Figure 3. Cutoff arguments of the $\mathbf{T}$ constant $\mathrm{S}_{\mathrm{n}}$ for perfectly conducting ground.

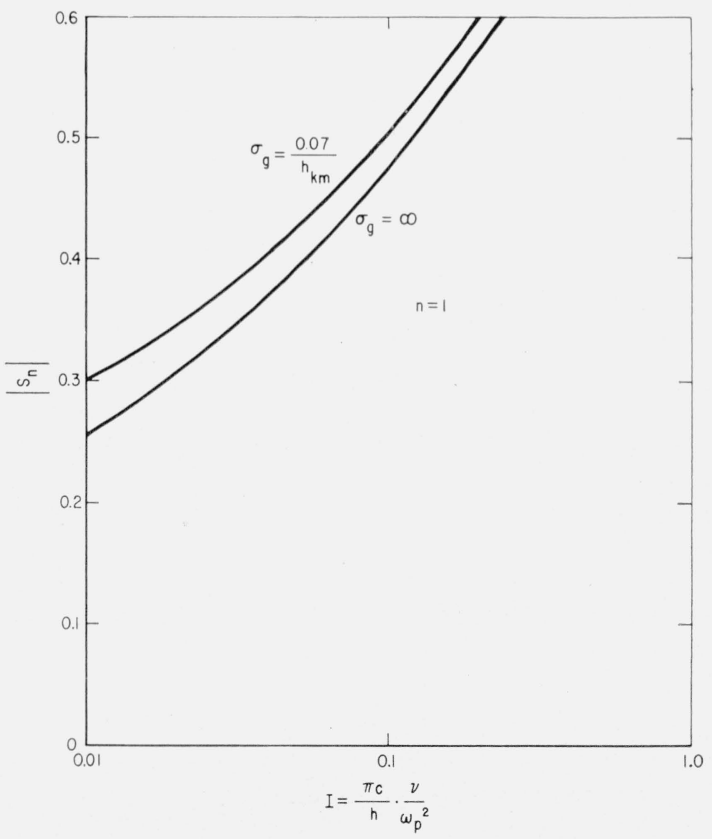

Figure 4. Magnitude of the constant $\mathrm{S}_{\mathbf{n}}$ near cutoff for the $\mathrm{n}=1$ mode. 


\section{Transient Fields}

The transient fields of the horizontal magnetic dipole may be computed as the Fourier integral of (32) for a specified spectrum of the antenna current. For a step of current the far field approximation (32) gives

$$
E_{r}^{h m}(\omega)=K \sqrt{i \omega} \sum_{n=0}^{\infty} \delta_{n} S_{n}^{0.5} e^{-i \frac{\omega d}{c} S_{n}}
$$

with

$$
K=\frac{\sin \phi I_{0} d a \eta}{2 h c \sqrt{2 \pi a c \sin (d / a)}}
$$

and with $\delta_{0}=0.5$ and $\delta_{n \neq 0}=1$.

The response of the $n=0$ mode may be reduced to tabulated Fourier integrals after approximating $S_{0}$ of $(52)$ by a power series. With $n_{g} \gg n_{i} \gg 1$ (45) reduces to

$$
S_{0} \approx \sqrt{1+\frac{1}{h \sqrt{i \omega \mu \sigma_{i}}}} .
$$

Expanding $\sqrt{S_{0}}$ and $S_{0}$ in powers of $(1 / \sqrt{i \omega})$ and ending the expansions with the quadratic terms results in

$$
E_{r}^{h m}(\omega)=K e^{d /\left(2 c \beta^{2}\right)}\left[\sqrt{i \omega}+\frac{1}{2 \beta}-\frac{3}{8 \beta^{2} \sqrt{i \omega}}\right] e^{-\frac{i \omega d}{c}-\frac{\sqrt{i \omega} d}{c \beta}},
$$

where

$$
\beta=2 h \sqrt{\mu \sigma_{i}}
$$

Applying the transform pairs (806), (801), and (807) of Campbell and Foster [1948] gives

$$
E_{r o}^{h m}(\tau) \approx \frac{K e^{1 / u-0.25 / x}}{2 \alpha^{1.5} \sqrt{\pi x}}\left[\frac{1}{2 x^{2}}-\frac{1}{x}\left(1-\frac{1}{u}\right)-\frac{3}{u^{2}}\right]
$$

where

$$
\begin{gathered}
x=\tau / \alpha, \\
\tau=(t-d / c)>0, \\
\alpha=\left(\frac{d}{\beta}\right)^{2}=\frac{\epsilon_{0} d^{2}}{4 \sigma_{i} h^{2}}=\frac{\nu d^{2}}{4 \omega_{p}^{2} h^{2}}, \\
u=\frac{2 c \beta^{2}}{d}=\frac{8 \sigma_{i} h^{2}}{d} \sqrt{\frac{\mu_{0}}{\epsilon_{0}}} .
\end{gathered}
$$

The power series expansion of (54) is applicable to frequencies where $\omega>\omega_{0}=\left(h^{2} \mu \sigma_{i}\right)^{-1}$. Hence (57) will be most accurate for $\tau<<2 \pi / \omega_{0}$ or for

$$
x<<x_{0}=2 \pi\left[\frac{2 h^{2} \sigma_{i}}{d} \sqrt{\frac{\mu_{0}}{\epsilon_{0}}}\right]^{2}=\pi u^{2} / 8 .
$$

Considering $h=90 \mathrm{~km}, d=10^{4} \mathrm{~km}, \sigma_{i}=10^{-6}$ mho $/ \mathrm{m}$ as an example, $x_{0}=2.3$ with $u=2.4$. The representation of the transient peak in figure 5 will be accurate even for this pessimistically low $\sigma_{i}$ value at $d=10^{4}$

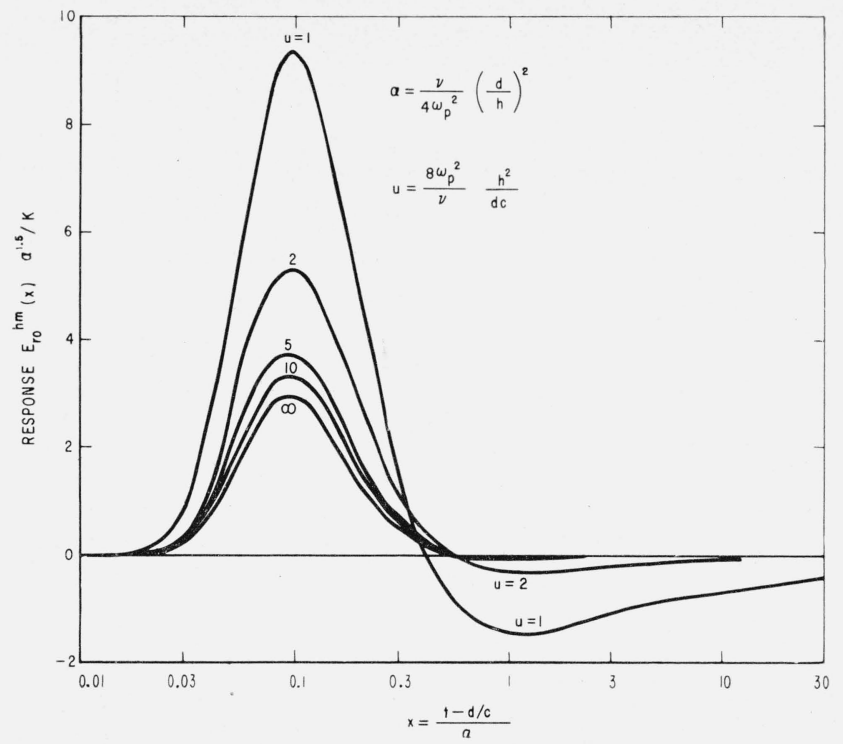

Figure 5. Response to a current step of the $\mathrm{n}=0$ mode of a horizontal magnetic dipole.

$\mathrm{km}$. Higher $\sigma_{i}$ and smaller $d$ result in larger $x_{0}$ (and $u$ ), which improves the accuracy of the transient tail representations.

It follows from reciprocity relation (3) that for equal current waveforms $I^{h m}(t)$ and $I^{v e}(t)$ in the $H M$ and $V E$ dipoles

$$
E_{r}^{h m}(t)=-\mu_{0} H_{\phi}^{v e}(t) \sin \phi d a / d s .
$$

A current in the $V E$ dipole that is proportional to the time derivative of the current in the $H M$ dipole

results in

$$
I^{v e}(t)=T I^{h m}(t),
$$

$$
E_{r}^{h m}(t)=-\mu_{0} \sin \phi d a H_{\phi}^{v e}(t) /(T d s) .
$$

The response $E_{r}^{h m}(t)$ to a current step $I_{0} u(t)$ should be proportional to the response $H_{\phi}^{v e}(t)$ to a current impulse $I_{0} T \delta(t)$. Assuming that (64) applies and assuming further that

$$
S_{0} \approx 1
$$

(this is obviously incorrect at the lower frequencies), it follows from (40) that

$$
E_{r}^{h m}(t) \approx-\sin \phi E_{r}^{v e}(t) d a /(c T d s) .
$$

The impulse response of a vertical electric dipole has been calculated considering factors proportional to finite powers of $S_{0}$ equal to unity [Schumann, 1952 ; Wait, 1960b]. The calculated impulse response of the $V E$ dipole [Schumann, 1952; Wait, 1960b] will be compared with the step response of the $H M$ dipole (57) by means of (65) and also by means of the approximate relation (67). The leading term 
of the impulse response (42) of Wait [1960b] (eqs (38), (39), (40), and (42) of Wait [1960b] should be multiplied by $\pi / 2$ ), when substituted in either (65) or (67) results in $E_{r}^{h m}(t)$ that is equal to (57) with the two terms proportional to $u^{-1}$ and $u^{-2}$ of the square brackets set equal to zero. There is no agreement between the higher response terms because of the $S_{0}$ approximation as discussed earlier. The impulse response of Schumann [1952], when substituted in (67) is the same as (57) with $u=\infty$.

The first order perturbation solution of the modal equation becomes inaccurate for the higher frequencies involved in calculating the transient response of the $n \neq 0$ modes. Even the simplest (and inaccurate) expressions of $S_{n}$ that may be obtained from (44) and (14) for $\left|n_{g}\right|>>\left|n_{i}\right|>>1$ involve integrals similar to those encountered in the transient analysis of lossy rectangular waveguides [Cerillo, 1948]. The transient response of the $n \neq 0$ modes has not been calculated in this paper.

\section{Appendix. Radial Electric Fields of Vertical Electric and Horizontal Magnetic Dipoles}

The fields of a vertical dipole may be derived from a single scalar function. Applying (29) of Schumann $[1954]$ to $(3)$

$$
\frac{E_{r}^{v e}}{E_{r}^{h m}}=\frac{d s_{1} \frac{\partial}{\partial \theta}\left(\sin \theta \frac{\partial u_{1}}{\partial \theta}\right)}{r k^{2} \cos \phi d a_{2} \sin \theta \frac{\partial u_{2}}{\partial \theta}}
$$

where for $u_{2}$ the coordinates of the source and of the observation points are interchanged relative to $u_{1}$. As long as the source and the observation point are approximately at the same radius $\left(r_{t} \approx r_{r}\right)$, both $u$ functions are the same. The ratio of the vertical electric fields of horizontal magnetic and vertical electric dipoles for equal currents on a perfectly conducting plane ground is

$$
\frac{E_{0}^{v e}}{E_{0}^{h m}}=\frac{i d s_{1}}{k d a_{2}} .
$$

With

$$
u_{1} \approx u_{2} \sim P_{\nu}(-\cos \theta)
$$

(68) becomes

$\frac{E_{r}^{v e}}{E_{r}^{h m}}=\frac{E_{0}^{v e}}{E_{0}^{h m} \cos \phi} \frac{i}{r k} \frac{(\nu+1) \sin \theta P_{\nu}(-\cos \theta)}{\cos \theta P_{\nu}(-\cos \theta)+P_{\nu-1}(-\cos \theta)}$.

The earlier approximations will reduce (71) to (41). However, more accurate $\nu$ values [Wait, 1960a, ch. $12]$ and a better approximation to $P_{\nu}(-\cos \theta)$ may be used in (71) to obtain a more exact $E^{v e} / E_{r}^{h m}$ ratio.

\section{References}

Bremmer, H., Terrestrial radio waves (Elsevier Publishing Co., New York, N.Y., 1949). See section III, 1 and 2.

Budden, K. G., The propagation of very low frequency radio waves to great distances, Phil. Nag. 44, 504 (1953).

Campbell, G. A., and R. M. Foster, Fourier integrals for practical applications (D. Van Nostrand Co., Inc., New York, N.Y., 1948).

Cerillo, M., Transient phenomena in waveguides, M.I.T. Research Lab. of Electronics, TR No. 33 (Jan. 3, 1948).

Howe, H. H., and J. R. Wait, Mode calculations for VLF ionospheric propagation, Proc. Symposium on Propagation of VLF Radio Waves 3, paper 36, Boulder, Colo. (1957).

Norton, K. A., The propagation of radio waves over the surface of the earth and in the upper atmosphere, pt. II, Proc. IRE 25, 1203 (1937)

Schumann, W. O., Über die Ausbreitung sehr langer elektrischer Wellen und der Blitzentladung um die Erde, Z. Angew. Phys. 4, 474 (1952).

Schumann, W. O., Über die Oberfelder bei der Ausbreitung langer, elektrischer Wellen im System Erde-Luft-Ionosphäre und 2 Anwendungen (horizontaler und senk-rechter Dipol), Z. Angew. Phys. 6, 35 (1954).

Sommerfeld, A., Partial differential equations in physics (Academic Press, Inc., New York, N.Y., 1949).

Wait, J. R., The mode theory of VLF ionosphere propagation for finite ground conductivity, Proc. IRE 45, 760 (1957).

Wait, J. R., Tertestrial propagation of VLF radio waves-a theoretical investigation, J. Research NBS 64D, 153 (1960a)

Wait, J. R., Vode theory and the propagation of ELF radio waves, J. Research NBS 64D, 387 (1960b).

Watson, G. N., The transmission of electric waves round the earth, Proc. Roy. Soc., Vol. A95, 546 (1919).

(Paper 65D3-133) 


\section{Publications of the National Bureau of Standards*}

\section{Selected Abstracts}

Amplitude-probability distributions for atmospheric radio noise, W. Q. Crichlow, A. D. Spaulding, C. J. Roubique, and R. T. Disney, NBS Mono. 23 (1960), 20 cents.

Families of amplitude-probability distribution curves are presented in a form such that by using three statistical parameters of atmospheric radio noise, of the type published by the National Bureau of Standards, the corresponding amplitude-probability distribution may be readily chosen. Typical values of these parameters are given.

Low- and very low-radiofrequency model ionosphere reflection coefficients, J. R. Johler, L. C. Walters, and J. D. Harper, Jr., NBS Tech. Note 69 (PB1615\%0), (July 1, 1960) $\$ 2.00$.

The results of extensive computations performed during the course of a theoretical investigation of a sharply bounded model ionosphere for low- and very low-radiofrequency wave propagation are presented in the form of graphs and tables. This Tech. Note supplements the work described in, On the theory of reflection of low- and very-low-radio frequency waves from the ionosphere, $J$. Research NBS 64D, No. 3 , 269 (May-June 1960).

The relation of $h_{\max } \mathrm{F} 2$ to $\mathrm{M}(3000) \mathrm{F} 2$ and $\boldsymbol{h}_{p} \mathrm{~F} 2, \mathrm{~J}$. W. Wright and R. E. McDuffie, J. Radio Research Laboratories 7, No. 32, 409 (July 1960)

Several easy methods of inferring directly from ionograms the height of the $F$ region peak, using the quantities $M(3000) F 2$ or $h_{p} F 2$, are compared with values of $h_{\max } F 2$ obtained from $N(h)$ profiles. It is found that such methods are free from bias at night at low and medium latitudes, but that systematic differences are significant in the daytime, and at high latitudes at all times. Examples are given illustrating the incorrect diurnal variation of $h_{\max } F 2$ estimated from these simple parameters, in comparison with simultaneous values of $h_{\max }$ obtained from $N(h)$ profiles.

A test of a procedure for easy estimation of representative monthly electron density profiles for the ionosphere, J. W. Wright, J. Geophys. Research 65, No. 10, 3215-3217 (Oct. 1960).

A recently proposed method for obtaining a representative monthly electron-density profile for a given hour of the day applies standard $N(h)$ procedures to a single representative virtual-height curve obtained in a special way from the individual daily virtual-height curves for that hour. The method is tested by comparing examples of the resulting profile with the average of the profiles for the individual days. The differences are small compared with the dispersion of the individual profiles about their mean. It is concluded that the method is valuable for obtaining a worldwide $N(h)$ morphology with a minimum expenditure of effort.

Spiral patterns in geophysics, V. Agy, J. Atmospheric and Terrest. Phys. 19, No. 2, 136-140 (Oct. 1960).

Recent analyses of magnetic and ionospheric data, notably by A. P. Nikolski, have resulted in spiral "precipitation" patterns which lead the authors to claim support for Störmer's theory of the aurora. Although it may be possible to argue against these claims by attacking the methods of analysis and/or the Störmer theory itself, an entirely different approach is used in the paper presented here: an examination of pertinent points of Störmer's theory shows that the analytical spirals mentioned cannot be Störmer spirals and this conclusion holds regardless of the soundness of the analyses and, indeed, of the validity of Störmer's theory.

Supplementary world maps of F2 critical frequencies and maximum usable frequency factors, Donald $H$. Zacharisen, NBS Tech. Note 2-2 (PB151361-2) (Oct. 1960) \$3.50.
This report supplements NBS Tech. Note 2 (April 1959), and completes the basic data required for F2-layer maximum usable frequency predictions. Prediction charts are given for the months of February, April, May, August, October, and November. Auxiliary charts are included to aid in predicting F2-layer MUFs.

The four parameters used for predicting MUFs are foF2 and the $4000 \mathrm{~km}$ MUF factor for a twelve-month running average Zurich sunspot number of 50 , and the rates of change of foF 2 and $4000 \mathrm{~km}$ MUF factor with sunspot number. The first three parameters are presented in map form for each even hour of Greenwich Mean Time. The fourth parameter is presented on a chart of geomagnetic latitude and local time.

The height of maximum luminosity in an auroral arc, F. E. Roach, J. G. Moore, E. C. Bruner, Jr., H. Cronin, and S. M. Silverman, J. Geophys. Research 65, No. 11, 3575-3580 (Nov. 1960).

The height of maximum luminosity of an auroral arc is estimated from simultaneous observations at three stations. in western United States during a night of general auroral activity (November 27-28, 1959). Photometrically this arc is characterized by a selective enhancement of the [OI] $6300 \mathrm{~A}$ line. From twenty-four individual measurements the height is found to be $412 \mathrm{~km}$ with a standard deviation of $\pm 23 \mathrm{~km}$ for one observation and $\pm 5 \mathrm{~km}$ for the mean. The geographical position of the arc, its orientation, and its movement during the night are discussed.

FM and SSB radiotelephone tests on a VHF ionospheric scatter link during multipath conditions, J. W. Koch, W. B. Harding, and R. J. Jansen, IRE Trans. Commun. Systems CS-8, No.3, 183-186 (Sept. 1960).

Experiments have been carried out on an ionospheric-scatter link to observe the effects of long-delayed multipath signals, caused by $F_{2}$ propagated back scatter, on the intelligibility of voice communication. Frequency modulation and singlesideband modulation equipments were used for the tests. During periods when the back-scatter signal levels approached the level of the normal ionospheric-scatter signals, the frequency-modulation voice transmissions were unintelligible; however, under the same conditions, single-sideband voice communication intelligibility remained at almost 100 per cent although there was some loss in quality.

Radio refractometry, Jack W. Herbstreit, NBS Tech. Note 66 (PB 161567) (July 1960) 50 cents.

The optical refractive index is known to be determined principally by the temperature and pressure of the atmosphere, whereas the radio refractive index is, in addition, affected by the water content of the atmosphere, the relationship between these quantities being expressed in the following way:

$$
N=(n-1) 10^{6}=\left(\frac{77.6}{T}\right)(P+[4810 e / T])
$$

where total air pressure $P$, and water vapor $e$, are in millibars, and the temperature $T$ is in degrees Kelvin [Smith, 1953]. The quantities $P, e$, and $T$ have long been routinely measured at the surface of the earth by standard weather bureau stations. For some time they have been measured from the surface up to great heights using balloon-borne radiosonde equipment at a large number of places over the earth's surface. More recently, equipment has been developed to measure rapidly and directly the radio refractive index of the atmosphere using radio techniques. The measurement of the radio refractive index properties of the atmosphere and the application to radio propagation problems is the subject of this paper. 


\section{Other NBS Publications}

Journal of Research, Vol. 65A, No. 2, March-April 1961. 70 cents.

Mass spectra of some deuteroethanes. Edith I. Quinn and Fred L. Mohler.

Heats of hydrolysis and formation of potassium borohydride. Walter H. Johnson, Richard H. Schumm, Isa H. Wilson, and Edward J. Prosen.

Heat of combustion of borazine $\mathrm{B}_{3} \mathrm{~N}_{3} \mathrm{H}_{6}$. Marthada V. Kilday, Walter H. Johnson, and Edward J. Prosen.

Thermodynamic properties of thorium dioxide from 298 to $1,200^{\circ} \mathrm{K}$. Andrew C. Victor and Thomas B. Douglas.

Calculated energy dissipation distribution in air by fast electrons from a gun source. John E. Crew.

Vitrons as flow units in alkali silicate binary glasses. Leroy W. Tilton.

Tetragermanates of strontium, lead, and barium of formula type $\mathrm{AB}_{4} \mathrm{O}_{9}$. Carl R. Robbins and Ernest M. Levin.

\section{Journal of Research, Vol. 65B, No. 1, January-March 1961.} 75 cents.

On transient solutions of the "baffled piston" problem. F. Oberhettinger

Special types of partitioned matrices. Emilie V. Haynsworth.

Bound for the $p$-condition number of matrices with positive roots. Philip J. Davis, Emilie V. Haynsworth, and Marvin Marcus.

Some computational problems involving integral matrices. Olga Taussky.

Computational problems concerning the Hilbert matrix. John Todd.

Index to the distributions of mathematical statistics. Frank A. Haight.

Selected bibliography of statistical literature, 1930 to 1957 IV. Markov chains and stochastic processes. Lola S. Deming and D. Gupta.

Building code requirements for reinforced masonry, NBS Handb. H74 (1960) 15 cents.

Amplitude, probability distributions for atmospheric radio noise, W. Q. Crichlow, A. D. Spaulding, C. J. Roubique, and R. T. Disney, N.BS Mono. 23 (1960) 20 cents.

Standard frequencies and time signals from NBS stations WWV and WWVH, NBS Misc. Pub 236 (1960) 10 cents.

Research highlights of the National Bureau of Standards, Annual Report, fiscal year 1960, NBS Misc. Pub. 237 (1960) 65 cents.

Quarterly radio noise data-December, January, February 1959-60, W. Q. Crichlow, R. D. Disney, and M. A. Jenkins, NBS TN18-5 (PB151377-5) (1960) $\$ 1.75$

Quarterly radio noise data-March, April, May 1960, W. Q. Crichlow, R. D. Disney, and M. A. Jenkins, NBS TN18-6 (PB151377-6) (1960) \$1.75.

On the nature of the crystal field approximation, H. Goldberg and C. Herzfeld, NBS TN67 (PB161568) (1960) $\$ 2.50$.

Vapor pressures of organic compounds in the range below one millimeter of mercury, E. E. Hughes and S. G. Lias, NBS TN70 (PB161571) (1960) 75 cents.

Some experiments on the deposition of gasses at $4.2^{\circ} \mathrm{K}, \mathrm{T}$. Baurer, NBS TN73 (PB161574) (1960) \$1.00.

Scattering of cobalt-60 gamma radiation in air duets, C. Eisenhauer, NBS TN74 (PB161575) (1960) 75 cents.

Soviet research in field electron and ion emission, 1955-59 an annotated bibliography, T. W. Marton and R. Klein, NBS TN75 (PB161576) (1960) \$1.25.

Oblique incidence receiving antenna array for a relative ionospheric opacity meter, A. C. Wilson, NBS TN78 (PB161579) (1960) 50 cents.

The dynamic compressibility of a rubber-sulfur vulcanizate and its relation to free volume, J. E. McKinney, H. V. Belcher, and R. S. Narvin, Trans. Soc. Rheology IV, 347 (1960)
Procedure for the determination of the noble metal content of dental gold alloys, H. J. Caul, W. S. Clabaugh and M. E. Susa, J. Am. Dental Assoc. 61, No. 4, 339 (Oct. 1960).

Theory of anisotropic fluids, J. L. Ericksen, Trans. Soc. Rheology IV, 29 (1960).

Rapid frequency analysis of fading radio signals, J. M. Watts and K. Davies, J. Geophys. Research 65, No. 8, 2295 (Aug. 1960)

On Stokes flow about a torus, W. H. Pell and L. E. Payne, Wathematika $\boldsymbol{7}, 78-92(1960)$

Reply to criticisms concerning Ireland contained in article, basic research in Europe, D. MI. Gates, Science 128, No. 3318, 1-9 (Aug. 1, 1958).

Thermal voltage converters for accurate voltage measurements to 30 megacycles per second, F. L. Hermach and E. S. Williams, Commun. Electron. AIEE, No. 49, 200 (July 1960).

Modern theories of materials, C. Truesdell, Trans. Soc. Rheology IV, 9 (1960)

Use of the incoherent scatter technique to obtain ionospheric temperatures, T. E. VanZandt and K. L. Bowles, J. Geophys. Research 65, 2627 (Sept. 1960).

Highlights of the consultative committee on international radio (CCIR) activities in the field of radio propagation, J. W. Herbstreit, 9th Plenary Assembly of CCIR Proc. IRE 48, No. 1, 45 (Jan. 1960).

Graphs for bivariate normal probabilities, M. Zelen and N. C. Severo, Ann. Math. Stat. 31, No. 3, 619 (Sept. 1960).

Temperature stratification in a non-venting liquid helium dewar, L. E. Scott, R. F. Robbins, and B. W. Birmingham, Proc. 1959 Cryogenic Engr. Conf. 5, (Sept. 2-4, 1959).

A microwave impedance meter capable of high accuracy, R. W. Beatty, IRE Trans. Microwave Theory and Tech. MTT-8, No. 4 (July 1960).

Variational treatment of electron-hydrogen atom elastic scattering, S. Geltman, Phys. Rev. 119, No. 4, 1283-1290 (Aug. 1960).

Relaxation processes in multistate systems, K. E. Shuler, Phys. Fluids 2, No. 4, 442 (July-Aug. 1959).

Comment on models of the ionosphere above $h_{\max } \mathrm{F} 2, \mathrm{~J}$. W. Wright, J. Geophys. Research 65, 2595 (Sept. 1960).

Some magnetoionic phenomena of the Artic E-region, J. W Wright, J. Atmospheric and Terrest. Phys. 18, 276 (Aug. 1960 ).

Tables of thermodynamic and transport properties of air, argon, carbon dioxide, carbon monoxide, hydrogen, nitrogen, oxygen and steam, J. Hilsenrath, C. W. Beckett, W. S. Benedict, L. Fano, H. J. Hoge, J. F. Masi, R. L Nuttall, Y. S. Touloukian, and H. W. Woolley, 478 pages (Pergamon Press, Oxford, London, New York, and Paris, 1960).

Methods of nuclear orientation, E. Ambler, Progress in Cryogenics 2, 235 (Heywood \& Co., Ltd., London, England, $1960)$.

A conference on the propagation of ELF electromagnetic waves, J. R. Wait, Proc. IRE 48, 1648 (Sept. 1960).

The strength of ten structural adhesives at temperature down to minus 424 F., W. M. Frost, Proc. 1959 Cryogenie, Engr. Conf. 5, 375 (Sept. 2-4, 1959).

The Stokes flow about a spindle, W. H. Pell and L. E. Payne, Quart. Appl. Math. 18, 257 (1960).

Possibility of detecting ionospheric drifts from the occurrence of spread $\mathrm{F}$ echoes at low latitudes, R. W. Knecht, Nature Letter 18\%, 927 (Sept. 1960).

A compilation and correlation of the PVT data of normal hydrogen from saturated liquid to 80 degree K., R. B. Steward and V. J. Johnson, Proc. 1959 Cryogenic Engr. Conf. 5, 548 (Sept. 2-4, 1959)

Pressure dependence of rotationally perturbed lines in the ultraviolet band spectrum of CN., H. P. Broida and S. Golden, Can. J. Chem. 38, 1666 (1960).

Neighbor interactions and internal rotations in polymer molecules. IV. Solvent effect on internal rotarions, S. Lifson and I. Openheim, J. Chem. Phys. 33, No. 1, 109 (July 1960).

Improvements in radio propagation prediction service, W. B. Chadwick, Elec. Engr. 1-4 (Sept. 1960).

Carbon resistance thermometry with mixed de and rf currents, 
J. J. Gniewek and R. J. Corruccini, Rev. Sci. Inst. 31, No. 8, 899 (Aug. 1960).

Some SEAC computations of subsonic flows, P. Davis and P. Rabinowitz, book, Bergman's Linear Integral Operator Method in the Theory of Compressible Fluid Flow, by M. Z. Krzywoblocki, p. 148 (Wien, Springer-Verlag, Berlin, Germany, 1960).

Spatial distribution of energy dissipated by fallout beta rays, A. E. Boyd and E. E. Morris, Health Phys. 2, 321 (1960). Maser frequency stability, R. C. Mockler and J. A. Barnes, Proc. 13 Annual Frequency Control Symp., 583 (May $12-14,1959)$.

Comment on a paper of Mori on time-correlation expressions for transport properties, M. S. Green, Phys. Rev. 119, No. 3, 829 (Aug. 1960)

Acid-base equilibria in benzene at three temperatures. The comparative reactivities of a phenolic acid and a carboxylic acid with triethylamine with 1, 3-diphenylguanidine, M. M. Davis and M. Paabo, J. Am. Chem. Soc. 82, 5081 (1960).

Absolute isotopic abundance ratio and the atomic weight of silver, W. R. Shields, D. N. Craig, and V. H. Dibeler, J. Am. Chem. Soc. 82, 5033 (1960).

Flexural strength of specimens prepared from several uranium dioxide powders; its dependency on porosity and grain size and the influence of additions of titania, F. P. Knudsen, H. S. Parker, and M. D. Burdick, J. Am. Ceram. Soc. 43, No. 12, 641 (Dec. 1960)

Green and purple sulfur: Electron-spin resonance studies, H. E. Radford and F. O. Rice, J. Chem. Phys. 33, No. 3, 774 (Sept. 1960).

Correlation of an auroral are with a subvisible monochromatic $6300 \mathrm{~A}$ arc with outerzone radiation on November 28, 1959, B. J. O'Brien, J. A. Van Allen, F. E. Roach, and C. W. Gartlein, J. Geophys. Research 65, No. 9, 2759 (Sept. 1960).

Report on the standardization of $\mathrm{pH}$ and related terminology, R. G. Bates and E. A. Guggenheim, Intern. Union Pure and Appl. Chem. 1, No. 1, 163 (1960).

Spectrum of $\mathrm{ReF}_{3}$, J. C. Eisenstein, J. Chem. Phys. 33, No. 5, 1530 (Nov. 1960).

Regulated power supply for instruments, W. V. Loebenstein, Electronics 33, No. 48, 132 (Nov. 1960).

Effect of structure on the spectra emitted by solid nitrogen during electron bombardment, L. J. Schoen and H. P. Broida, J. Mol. Spectroscopy 5, No. 5, 416 (Nov. 1960).

The compound $\mathrm{BaTiGe}_{3} \mathrm{O}_{9}, \mathrm{C}$. R. Robbins, J. Am. Ceram. Soc. 43, No. 11, 610 (Nov. 1960).

Chemical reactions of free radicals at low temperatures, R. A. Ruehrwein, J. S. Hashman and J. W. Edwards, J. Phys. Chem. 64, 1317 (1960).

Experimental investigation of creep deflection of extruded and riveted I-beams, L. Mordfin and N. Halsey, NASA Tech. Note D-662 (Dec. 1960).

Spatial distribution of energy dissipated by fallout-rays, A. E. Boyd and E. E. Morris, Health Phys. 2, 321 (1959).

Megaroentgen dosimetry employing photographic film without processing, W. L. McLaughlin, Radiation Research 13, No. 4, 594 (Oct. 1960).

The reaction of hydrogen atoms with solid propene at low temperatures, R. Klein, M. D. Scheer and J. G. Waller, J. Phys. Chem. 64, 1247 (1960).

The measurement of thermal conductivity, D. C. Ginnings, Book, Thermoelectric Materials and Devices, edited by I. B. Cadoff and E. Miller. Chapter 8, 113 (Reinhold Publ. Corp., New York, N.Y., 1960).
Programming for a closed-loop, manned-machine combined system, D. C. Friedman, Proc. Combined Analog-Digital Computer Systems Symp., December 16 and 17, 1960, Philadelphia, Pa., 12th Article (Dec. 1960).

Irrational power series, M. Newman, Proc. Am. Math. Soc. 11, 699 (Oct. 1960)

Analytical study of creep deflection of structural beams, L. Mordfin, NASA Tech. Note D-661 (Dec. 1960).

Evaluation of ball bearing separator materials operating submerged in liquid nitrogen, W. A. Wilson, K. B. Martin, J. A. Brennan, and B. W. Birmingham, ASLE. ASME Lubrication Conf., October 17-19, 1960 (Boston, Mass.), Am. Soc. Lubrication Engrs. Preprint No. 60 LC-4 (1960).

Microwave spectrum, structure, and dipole moment of propane, D. R. Lide, Jr., J. Chem. Phys. 33, No. 5, 1514 (Nov. 1960).

Topological derivation of the Mayer density series for the pressure of an imperfect gas, M. S. Green, J. Math. Phys. 1, No. 5, 391 (Sept.-Oct. 1960).

Surface area determination of kaolinite using glycerol adsorption, K. H. Woodside and W. C. Ormsby, J. Am. Ceram. Soc. 43, No. 12, 671 (Dec. 1960).

Magnetic resonance determination of the nuclear moment of tantalum-181 in $\mathrm{KTaO}_{3}$, L. H. Bennett and J. I. Budnick, Phys. Rev. 120, No. 5, 1812 (Dec. 1, 1960).

Aircraft storage batteries, W. J. Hamer, AIEE and Am. Inst. Elec. Engr. Trans. 79, Pt. II, 1-11 (Sept. 1960).

Electrodeless passage of direct current through an electrolyte, A. Brenner, J. Electrochem. Soc. 10\%, No. 12, 968 (Dec. 1960).

Preparation and thermal stability of tetrakis-(pentafluorophenyl)-silane and tris-(pentafluorophenyl)-phosphine, L. A. Wall, R. E. Donadio, and W. J. Pummer, J. Am. Chem. Soc. 82, No. 18, 4846 (Sept. 1960).

Stress-strain relationships in yarns subjected to rapid impact loading. Part VI: Velocities of strain waves resulting from impact, J. C. Smith, J. M. Blanford, and H. F. Schiefer, Textile Research J. 30, No. 10, 752 (Oct. 1960). The centennial of spectrochemistry, W. F. Meggers, J. L. Tech, J. Opt. Soc. Am. 50, No. 11, 1035 (Nov. 1960).

Rate of reaction of nitrogen atoms with ethylene, J. T. Herron, J. Chem. Phys. 33, No. 4, 1273 (Oct. 1960).

Structure of the isobutane molecule; change of dipole rnoment on isotopic substitution, D. R. Lide, Jr., J. Chem. Phys. 33, No. 5, 1519 (Nov. 1960).

Condensation coefficient of arsenic trioxide glass, A. B. Bestul and D. H. Blackburn, J. Chem. Phys. 33, No. 4, 1274 (Oct. 1960).

Some mechanical properties of magnesium alloys at low temperatures, R. P. Reed, R. P. Mikesell and R. L. Greeson, Proc. 1959, Cryogenia Eng. Conf. 5, 397 (Sept. 2-4, 1959).

Electron spin resonance studies of free radicals in irradiated materials, L. A. Wall, Symp. on Materials in Nuclear Application, Am. Soc. Testing Materials Spec. Tech. Publ. No. 276 , p. 208 (1959).

* Publications for which a price is indicated (except for Technical Notes) are available only from the Superintendent of Documents, U.S. Government Printing Office, Washington 25, D.C. (foreign postage, one-fourth additional). The Technical News Bulletin and Basic Radio Propagation Predictions are available on a 1-, 2-, or 3-year subscription basis, although no reduction in rates can be made. Reprints from outside ournals and the NBS Journal of Research may often be obtained directly from the authors. 